\title{
Corrections to: Molluscicidal effectiveness of Luo-Wei, a novel plant-derived molluscicide, against Oncomelania hupensis, Biomphalaria alexandrina and Bulinus truncatus
}

Tie-Wu Jia ${ }^{1,2,3,4,5,6+}$, Wei Wang ${ }^{7 \dagger}$, Le-Ping Sun 7 , Shan Lv1,2,3,4,5 Kun Yang $^{7}$, Neng-Min Zhang ${ }^{8}$, Xi-Bao Huang ${ }^{9}$, Jian-Bing Liu', Han-Cheng Liu', Rui-Hua Liư ${ }^{10}$, Fathia A. Gawish ${ }^{11}$, Mohamed R. Habib ${ }^{11}$, Mohamed A. El-Emam" Charles H. King ${ }^{12,13^{*}}$ and Xiao-Nong Zhou ${ }^{1,2,3,4,5^{*}}$

Correction to: Infectious Diseases of Poverty (2019) 8:27 https://doi.org/10.1186/s40249-019-0535-7

In the abstract of original publication of the article [1], $0.33 \mathrm{mg} / \mathrm{L}\left(24 \mathrm{~h} \mathrm{LC}_{50}\right.$ against $B$. alexandrina) should be replaced by $1.975 \mathrm{mg} / \mathrm{L}$ which was clearly showed in Table 1 . We regret any confusion this error may have caused. The original publication has been corrected.

\footnotetext{
Author details

${ }^{1}$ National Institute of Parasitic Diseases, Chinese Center for Disease Control and Prevention, Shanghai 200025, China. ${ }^{2}$ Chinese Center for Tropical Diseases Research, Shanghai 200025, China. ${ }^{3}$ WHO Collaborating Centre for Tropical Diseases, Shanghai 200025, China. ${ }^{4}$ National Center for International Research on Tropical Diseases, Ministry of Science and Technology, Shanghai 200025, China. ${ }^{5}$ Key Laboratory of Parasite and Vector Biology, Ministry of Health, Shanghai 200025, China. ${ }^{6}$ Communicable Diseases Cluster, World Health Organization Regional Office for Africa (WHO/AFRO), PO Box 06, Brazzaville, Congo. 'Key Laboratory of National Health Commission on Parasitic Disease Control and Prevention, Jiangsu Provincial Key Laboratory on Parasites and Vector Control Technology, Jiangsu Institute of Parasitic Diseases, Wuxi 214064, China. ${ }^{8}$ Hubei Jinhaichao Science \& Technology Co.,Ltd, Wuhan 430206, China. ${ }^{9}$ Hubei Provincial Center for Disease Control and Prevention, Wuhan 430079, China. ${ }^{10}$ School of Chemistry and

ChemicalEngineering, Wuhan Textile University, Wuhan 430200, China. ${ }^{11}$ Department of Medical Malacology, Theodor Bilharz Research Institute (TBRI), Imbaba, Giza 12411, Egypt. ${ }^{12}$ Center for Global Health and Diseases, Case Western Reserve University, Cleveland, OH, USA. ${ }^{13}$ Schistosomiasis Consortium for Operational Research and Evaluation, University of Georgia, Athens, GA, USA.
}

Received: 9 May 2019 Accepted: 9 May 2019

Published online: 06 June 2019

\section{Reference}

1. Jia, et al. Molluscicidal effectiveness of Luo-Wei, a novel plant-derived molluscicide, against Oncomelania hupensis, Biomphalaria alexandrina and Bulinus truncatus. Infectious Diseases of Poverty. 2019;8:27 https://doi.org/ 10.1186/s40249-019-0535-7.

\footnotetext{
* Correspondence: chk@case.edu; xiaonongzhou1962@gmail.com

${ }^{\dagger}$ Tie-Wu Jia and Wei Wang contributed equally to this work.

${ }^{12}$ Center for Global Health and Diseases, Case Western Reserve University, Cleveland, OH, USA

${ }^{1}$ National Institute of Parasitic Diseases, Chinese Center for Disease Control and Prevention, Shanghai 200025, China
} 